\title{
A Randomized Observer-Blinded Controlled Trial to Compare Pre-Emptive with Postoperative Ultrasound-Guided Mandibular Nerve Block for Postoperative Analgesia in Mandibular Fracture Surgeries
}

This article was published in the following Dove Press journal: Local and Regional Anesthesia

\section{Rajagopalan Venkatraman (I) Kandhan Karthik Cherian Belinda (D) Ramamurthy Balaji}

Department of Anaesthesia, SRM Medical College Hospital and Research Centre, Potheri, Tamilnadu, 603203, India

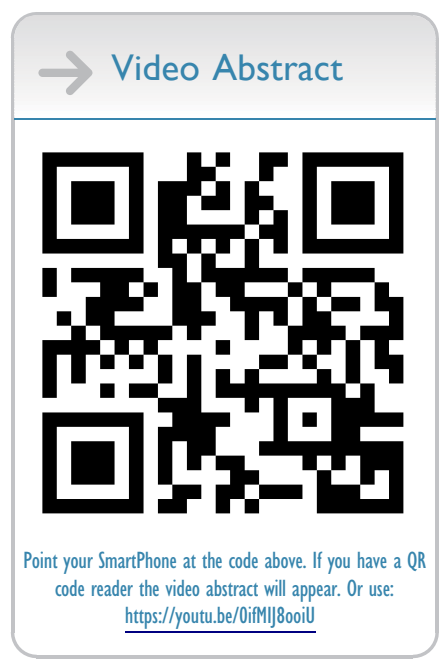

Correspondence: Rajagopalan Venkatraman Department of Anaesthesia, SRM Medical College Hospital and Research Centre,

Potheri, 603203, Tamilnadu, India

Tel +9l 989458I 455

Email drvenkat94@gmail.com
Background and Aims: Ultrasound-guided (UG) mandibular nerve block is effective for providing postoperative analgesia in mandibular fracture surgeries. The pre-emptive nerve blockade prolongs the duration of postoperative analgesia and reduces the consumption of intraoperative opioids. The aim of this prospective, randomized, single-blinded study was to compare the efficacy of pre-emptive and postoperative UG mandibular nerve block for postoperative analgesia in mandibular fracture surgeries.

Methods: Sixty patients scheduled for unilateral mandibular fracture surgeries were randomly divided into two groups by computer-generated random numbers and sealed envelope method: Group A received UG mandibular nerve block before surgical incision and group B received after surgery with ropivacaine $0.5 \% 10 \mathrm{~mL}$. The second anesthesiologist, who was blinded to the group involved, monitored the patient. The patients as well as the statistician were also blinded. The patients were started on patient-controlled analgesia (PCA) morphine with bolus $1 \mathrm{mg}$ and a lockout interval of $10 \mathrm{~min}$. The morphine consumption for $24 \mathrm{~h}$ was recorded. The pain was assessed by the VAS score. The additional intraoperative fentanyl consumption and time for a request for rescue analgesic were recorded.

Results: The total morphine consumption was reduced in group A $(4.566 \pm 0.717 \mathrm{mg})$ than group B $(5.93 \pm 0.876 \mathrm{mg})$ with a p-value of $<0.0001$. The time for a request for rescue analgesic was also prolonged in group A (794.08 $\pm 89.561 \mathrm{~min})$ than group B (505.333 $\pm 3.159 \mathrm{~min}$ ). In group $\mathrm{A}$, only four patients required an additional dose of fentanyl as against 11 patients in group B. The heart rate was also lower in group A 30min after the administration of the block and persisted for two hours intraoperatively.

Conclusion: Pre-emptive ultrasound-guided mandibular nerve block reduces morphine consumption, prolongs the time for a request for rescue analgesic, reduces intraoperative fentanyl consumption, provides better control of intraoperative heart rate, and better pain scores postoperatively when compared to the postoperative mandibular nerve block.

Keywords: mandibular fracture, mandibular nerve, postoperative pain, ultrasound-guided, visual analog scale

\section{Introduction}

The surgery for mandibular fracture fixation can cause severe pain in the immediate postoperative period. Most of these patients are managed with intravenous opioids. 
These may have side-effects like nausea, vomiting, pruritus, and respiratory depression. ${ }^{1,2}$ Mandibular nerve block provides good perioperative analgesia for mandibular and intra-oral surgeries. They can be used as part of multimodal analgesia to reduce the consumption of opioids in the postoperative period.

The purpose of pre-emptive analgesia is not only to provide better postoperative analgesia but also to reduce the consumption of opioids and anesthetic agents intraoperatively. ${ }^{3}$ The pre-emptive analgesia was found to be effective in reducing the incidence of the development of chronic pain. ${ }^{4}$ Several studies have proved the efficacy of pre-emptive analgesia in reducing the consumption of opioids postoperatively. ${ }^{5,6}$ However, some studies and a systematic review have demonstrated that pre-emptive analgesia is not beneficial. ${ }^{7}$

No studies are showing the efficacy of ultrasoundguided (UG) mandibular nerve block for mandibular fracture surgeries to the best of our knowledge. Our study aimed to compare the consumption of morphine for 24 hours (h) postoperatively between pre-emptive and postoperative UG mandibular nerve block for mandibular fracture surgeries. The secondary objectives were to compare the time for a request for rescue analgesic, intraoperative hemodynamic parameters, additional fentanyl consumption, and visual analog scale (VAS) scores for $24 \mathrm{~h}$ postoperatively.

\section{Methods}

After obtaining approval from SRM Medical College Hospital and Research Centre ethical committee, this prospective, randomized, single-blinded study was prospectively registered in Clinical Trial Registry - India (CTRI/ 2019/11/021902) and following good clinical practice and the guidelines set out in the Declaration of Helsinki. Patients with American Society of Anesthesiologists' (ASA) physical status I and II between 18 and 70 years of age, scheduled for unilateral mandibular fracture surgeries under general anesthesia from November 2019 to September 2020 at SRM Medical College Hospital and Research Centre, Chennai, India were included in the study. Exclusion criteria included: fracture of symphysis; pregnant patients; who are allergic to local anesthetics (LA); coagulation abnormalities; chronic cardiac, hepatic, or renal diseases; or who refuse to participate in the study.

At the time of pre-operative assessment, patients were informed about the study protocol, explained about the VAS score and written consent was obtained. The randomization was performed using computer-generated random numbers and placed in a sealed, opaque envelope. At the start of the surgery, the envelope was opened by the anesthesiologist administering the case and allocated to one of the two groups. Sixty patients were randomly allocated into two groups: group A patients receiving UG mandibular nerve block after administration of general anesthesia and before surgical incision and group B patients receiving UG mandibular nerve block at the end of the surgery, before extubation.

General anesthesia was standardized in both groups. All the patients were premedicated with alprazolam $0.5 \mathrm{mg}$ orally on the night and two hours before surgery. The patients were monitored with a pulse oximeter, noninvasive blood pressure, electrocardiograph, and capnography intraoperatively. Fentanyl $2 \mathrm{mcg} / \mathrm{kg}$ was administered before induction and repeated at one-third of the initial dose if there was an unexplained increase in heart rate or mean arterial pressure by more than $20 \%$. The number of patients requiring additional bolus doses of fentanyl was recorded. The patients were induced with propofol $2 \mathrm{mg} / \mathrm{kg}$ and vecuronium was used as a muscle relaxant. Anesthesia was maintained with air oxygen mixture and sevoflurane. In group A patients, the mandibular nerve block was administered after endotracheal intubation and after surgery in group B patients.

An Ultrasonogram (USG) Machine (Logiq V2, GE Medical Systems, Jiangsu, China), with a 5-13MHz linear probe was used. The patient was placed in the supine position with the head turned to the opposite side. Under strict aseptic precautions, the ultrasound probe was placed below and parallel to the zygomatic arch. The perimandibular space (PMS) was identified between the coronoid process and the condyle of the mandible (Figure 1). Using an out of the plane technique from the cranial end, the needle was targeted around the maxillary artery and $10 \mathrm{~mL}$ of $0.5 \%$ ropivacaine is administered. All the vital parameters were recorded every 15 minutes (min) till the completion of surgery. At the end of the surgery, the patient was extubated after reversal with neostigmine and glycopyrrolate.

The patient was shifted to the post-anesthetic care unit (PACU) and managed by another anesthesiologist who was blinded to the group involved. The patients were monitored for heart rate, blood pressure, and oxygen saturation for $24 \mathrm{~h}$. VAS score was used to assess pain where 0 is no pain and 10 as the worst pain (Figure 2). ${ }^{8}$ The patient was started on patient control analgesia with morphine. The bolus dose was kept at $1 \mathrm{mg}$ with 10 minutes as a lockout interval and 


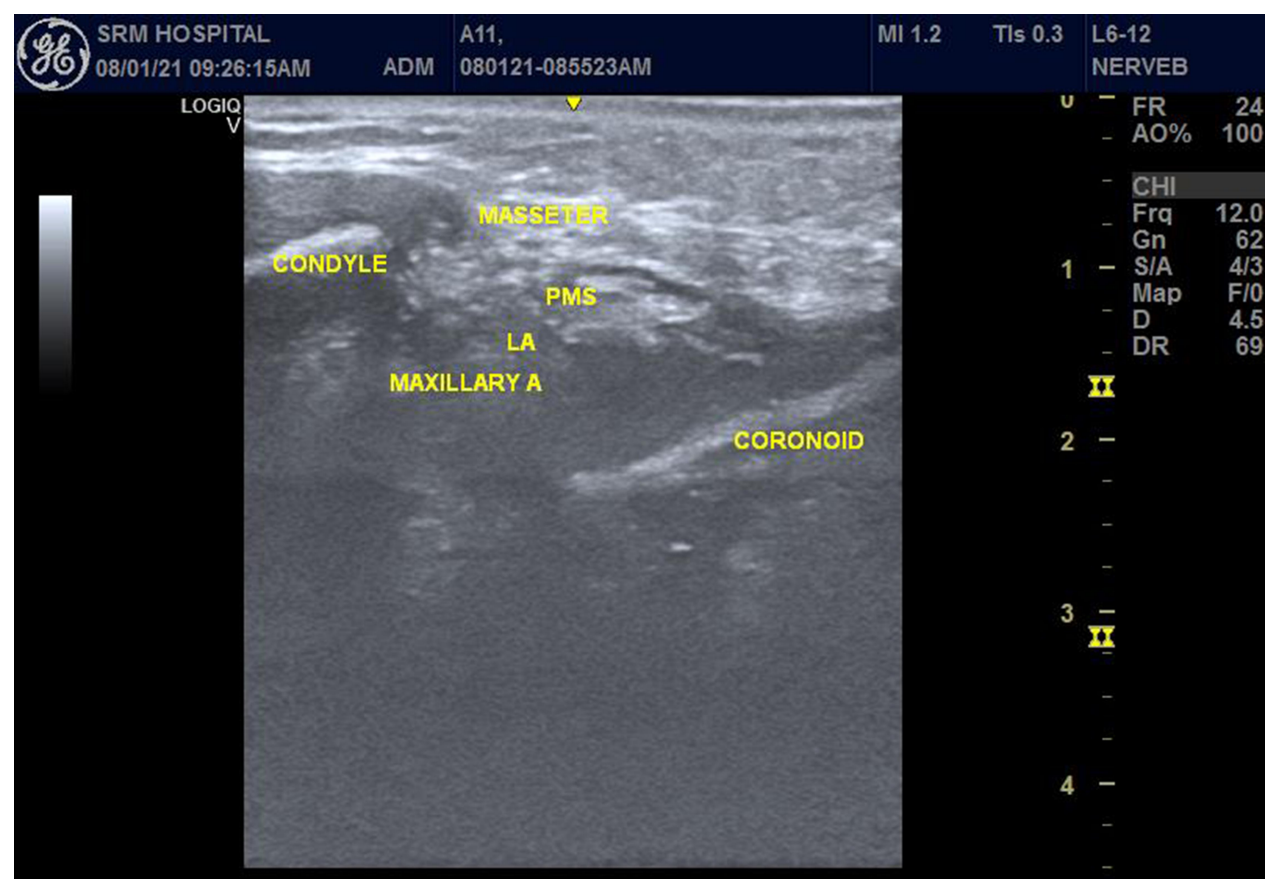

Figure I Ultrasound-guided mandibular nerve block.

a baseline infusion of $0.1 \mathrm{mg} / \mathrm{h}$. The patient was instructed to use a bolus dose when VAS $\geq 3$. The total consumption of morphine during the first postoperative $24 \mathrm{~h}$ was recorded. The time for a request for rescue analgesic was taken as the time consumed from extubation to the first bolus consumption of morphine. The VAS scores were compared every four hours for $24 \mathrm{~h}$. The patients were observed for any adverse effects like nausea, vomiting, pruritus, urinary retention, hypotension, and bradycardia.

The sample size was estimated based on a pilot study of 10 patients for each group. The mean difference in the total consumption of morphine was $3.16 \mathrm{mg}$ and the pooled standard deviation was $5 \mathrm{mg}$. For the study to have $80 \%$ power and alpha error at 0.05 , a minimum of 27 patients would be required in each group to detect a $20 \%$ difference in the consumption of morphine between the two groups. We recruited 30 patients in each group to compensate for the possible dropouts. The statistical analysis was performed using Statistical Package for Social Sciences version 20.0 software (IBM Corp., Armonk, New York, USA). The normal distribution was checked by the Kolmogorov-Smirnov test. The categorical variables were expressed as the number of patients (percentage) and compared using Fisher's exact and Chi-square test. The continuous variables were expressed as mean \pm standard deviation and compared using an unpaired $t$-test. A P-value $<0.05$ was considered to indicate the statistical significance and $<0.0001$ as extremely statistically significant.

\section{Results}

Sixty patients were recruited for the trial and no patient was lost to follow-up. The flow diagram of the progress through the phases of the trial was depicted in the

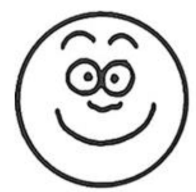

0

No Hurt

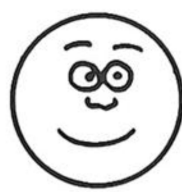

2

Hurts Little Bit

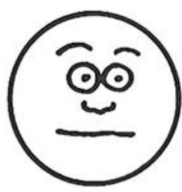

4

Hurts Little More

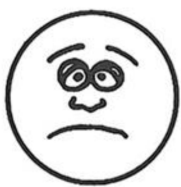

6

Hurts Even More

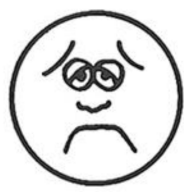

8

Hurts

Whole Lot

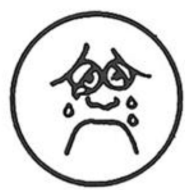

10

Hurts

Worst

Figure 2 Visual analog scale (VAS). 
Consolidated Standards of Reporting Trials (CONSORT) (Figure 3). The demographic characteristics of the studied population were given in Table 1. There was no difference in age, sex, ASA physical status, parts of mandibular fracture, and duration of surgery between the two groups.

The total morphine consumption was $4.566 \pm$ $0.717 \mathrm{mg}$ in group A and $5.93 \pm 0.876 \mathrm{mg}$ in group B. it was statistically highly significant with a p-value of $<$ 0.0001 . The time for request for a rescue analgesic was significantly prolonged in group A with $794.08 \pm 89.561$ min than group B with $505.333 \pm 73.159 \mathrm{~min}$ (p-value of $<0.0001)$. The intraoperative fentanyl consumption was significantly reduced in group A with only four patients requiring it. In group $\mathrm{B}, 11$ patients required additional doses. It was statistically significant with a p-value of 0.037. The values were summarized in Table 2. There were no variations in heart rate for the first 30 minutes of surgery. After the first 30 minutes, the heart rate was significantly less in group A than group B (Table 3). There were no statistically significant variations in intraoperative mean arterial pressure between the two groups (Table 4). There was a statistically significant reduction in VAS scores in group A than group B from eight hours (h) to $20 \mathrm{~h}$ postoperatively (Figure 4 ).

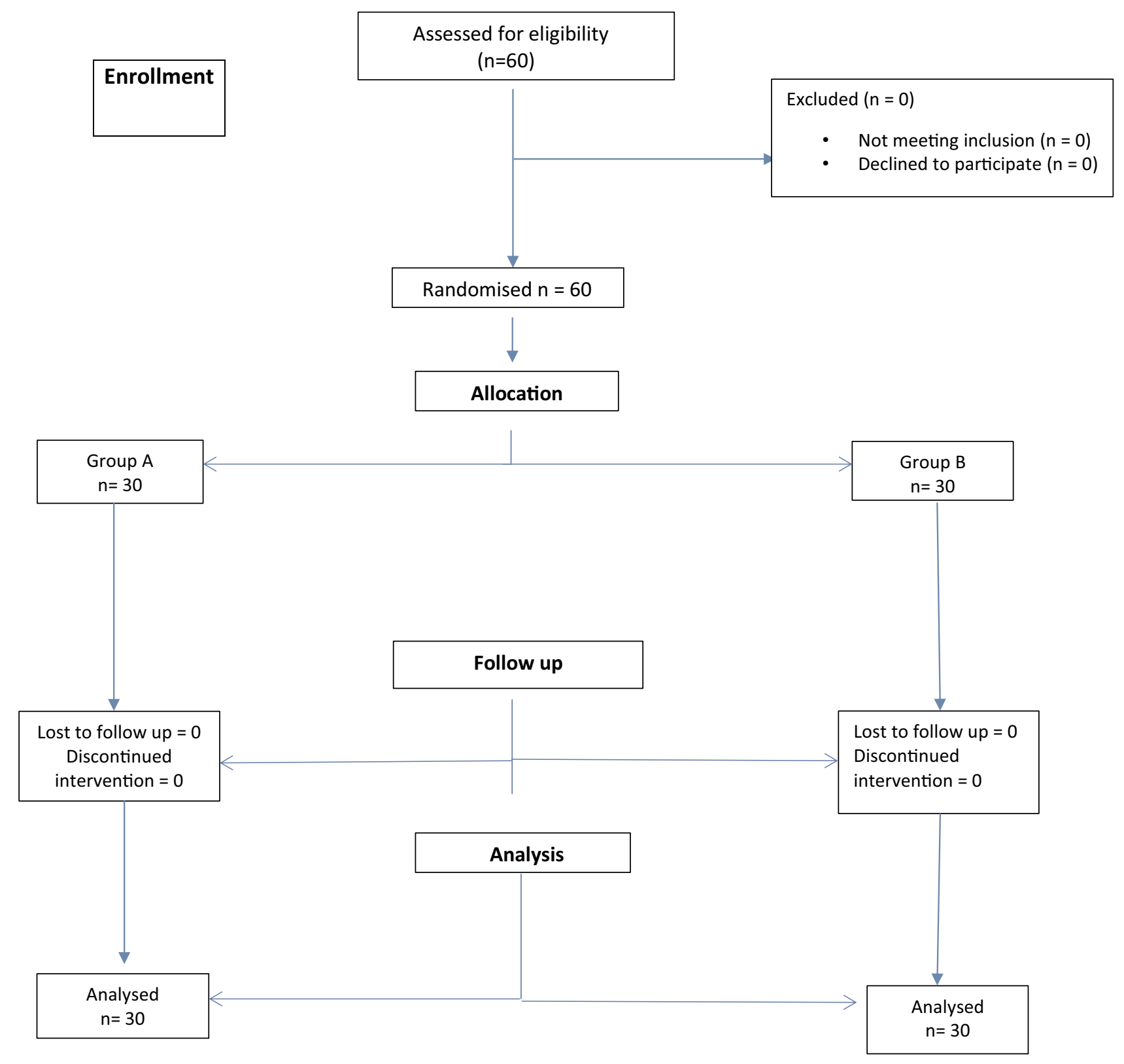

Figure 3 Consolidated Standards of Reporting Trials (CONSORT) flow chart. 
Table I Demographic Data

\begin{tabular}{|c|c|c|c|}
\hline Parameters & Group A & Group B & P-value \\
\hline Age & $34.066 \pm 5.353$ & $32.060 \pm 4.094$ & $0.108^{*}$ \\
\hline Duration of surgery (min) & $98.17 \pm 11.795$ & $95 \pm 3.57$ & $0.162 *$ \\
\hline Gender (M/F) & $20 / 10$ & $16 / 14$ & $0.625^{*}$ \\
\hline ASA $(I / I I)$ & $24 / 6$ & $22 / 8$ & $0.373 *$ \\
\hline Mandibular part fracture (Body/Angle/Ramus/Condyle) & $8 / 9 / 1 / 12$ & $10 / 8 / 1 / 11$ & $0.324 *$ \\
\hline
\end{tabular}

Notes: Values are in Mean \pm SD or number of patients. ${ }^{*} \mathrm{p}$-value not significant (unpaired $t$-test, Chi-square test).

Table 2 Anaesthetic Data

\begin{tabular}{|l|c|c|c|}
\hline Parameters & Group A & Group B & P-value \\
\hline Total morphine consumption (mg) & $4.566 \pm 0.72$ & $5.93 \pm 0.87$ & $<0.000 \mathrm{I}^{\mathrm{T}}$ \\
Duration of post op analgesia (min) & $794.08 \pm 89.56$ & $505.33 \pm 73.15$ & $<0.000 \mathrm{I}^{\mathrm{T}}$ \\
Additional fentanyl consumption & $4(\mathrm{I} 3.3 \%)$ & $\mathrm{II}(36.7)$ & $0.037^{\mathrm{T}}$ \\
\hline
\end{tabular}

Notes: Values are in Mean \pm SD or number of patients (percentage). ${ }^{\mathrm{T}} \mathrm{p}$-value significant (unpaired $t$-test, Chi-square test).

Table 3 Perioperative Heart Rate

\begin{tabular}{|l|c|c|c|}
\hline Time in Min & Group A & Group B & P-value \\
\hline Baseline & $84.76 \pm 4.90$ & $86.20 \pm 5.12$ & $0.272^{*}$ \\
15 & $83.26 \pm 3.69$ & $81.43 \pm 5.08$ & $0.115^{*}$ \\
30 & $72.70 \pm 3.88$ & $74.96 \pm 5.45$ & $0.069^{*}$ \\
45 & $71.13 \pm 3.59$ & $74.63 \pm 5.21$ & $0.003^{T}$ \\
60 & $68.96 \pm 4.05$ & $73.3 \pm 3.75$ & $<0.0001^{\mp}$ \\
75 & $68.06 \pm 3.44$ & $71.66 \pm 2.99$ & $0.0004^{\mp}$ \\
90 & $69.26 \pm 4.63$ & $74.4 \pm 2.53$ & $<0.0001^{\mp}$ \\
105 & $71.5 \pm 6.1$ & $74.2 \pm 3.3$ & $0.037^{\mp}$ \\
120 & $70.63 \pm 5.55$ & $74.06 \pm 2.56$ & $0.003^{\mp}$ \\
\hline
\end{tabular}

Notes: Values are in Mean $\pm \mathrm{SD}$. ${ }^{*} \mathrm{p}$-value not significant (unpaired $t$-test). ${ }^{\mathrm{t}} \mathrm{p}$-value significant.

Table 4 Perioperative Mean Arterial Pressure

\begin{tabular}{|l|c|c|c|}
\hline Time in Min & Group A & Group B & P-value \\
\hline Baseline & $92.36 \pm 3.42$ & $93.53 \pm 3.04$ & $0.168^{*}$ \\
15 & $79.9 \pm 4.35$ & $80.96 \pm 3.48$ & $0.299^{*}$ \\
30 & $77.4 \pm 3.44$ & $78.5 \pm 3.63$ & $0.233^{*}$ \\
45 & $74.56 \pm 2.36$ & $75.43 \pm 2.71$ & $0.949^{*}$ \\
60 & $73.93 \pm 1.89$ & $74.1 \pm 1.77$ & $0.726^{*}$ \\
75 & $74.03 \pm 2.65$ & $74.2 \pm 1.79$ & $0.772^{*}$ \\
90 & $76.83 \pm 4.09$ & $77.52 \pm 3.37$ & $0.493^{*}$ \\
105 & $78.26 \pm 4.2$ & $78.76 \pm 3.35$ & $0.612^{*}$ \\
120 & $77.63 \pm 3.90$ & $78.46 \pm 3.77$ & $0.402^{*}$ \\
\hline
\end{tabular}

Notes: Values are in Mean $\pm \mathrm{SD}$. *p-value not significant (unpaired $t$-test).

\section{Discussion}

Pre-emptive analgesia was defined as the preoperative administration of analgesia before the surgical stimulus and prevents the establishment of central sensitization due to incisional as well as inflammatory injury. ${ }^{9}$ The concept of pre-emptive analgesia to attenuate the severity and prolong the duration of postoperative pain was described earlier by $1983 .{ }^{10}$ There have been conflicting reports regarding the efficacy of pre-emptive analgesia with few studies supporting ${ }^{7,11-13}$ while few refuting the efficacy. ${ }^{13,14}$ Pre-emptive analgesia was believed to prevent the processing of afferent input, which usually increases the postoperative pain. Although clinical studies have failed to establish the efficacy of Pre-emptive analgesia, it was important to take inflammatory injury into concern. ${ }^{15}$ The failure of a few studies may be due to the initiation of Pre-emptive analgesia after the inflammatory injury. ${ }^{7,15}$ Preemptive analgesia continues to have promise for the effective treatment of postoperative pain.

The mandibular nerve, branch of the trigeminal nerve, is a mixed sensory and motor nerve. The mandibular nerve exits the cranium through the foramen ovale that supplies the front of the ear, the temporal area, the anterior two-thirds of the tongue, and the skin, mucosa, and teeth, and bone of the mandible. The mandibular nerve is blocked where the nerve exits the foramen ovale. Complete block results in anesthesia of the ipsilateral mandibular bone, lower teeth up to the midline, buccal and lingual hard and soft tissue, anterior two-thirds of the tongue, the floor of the mouth, the external acoustic meatus, and auricle of the ear in its anterior zone, the skin over the jaw, the posterior part of the cheek, and the temporal area (except the area of the angle of the mandible, which is supplied by the superficial cervical plexus). ${ }^{16}$ 


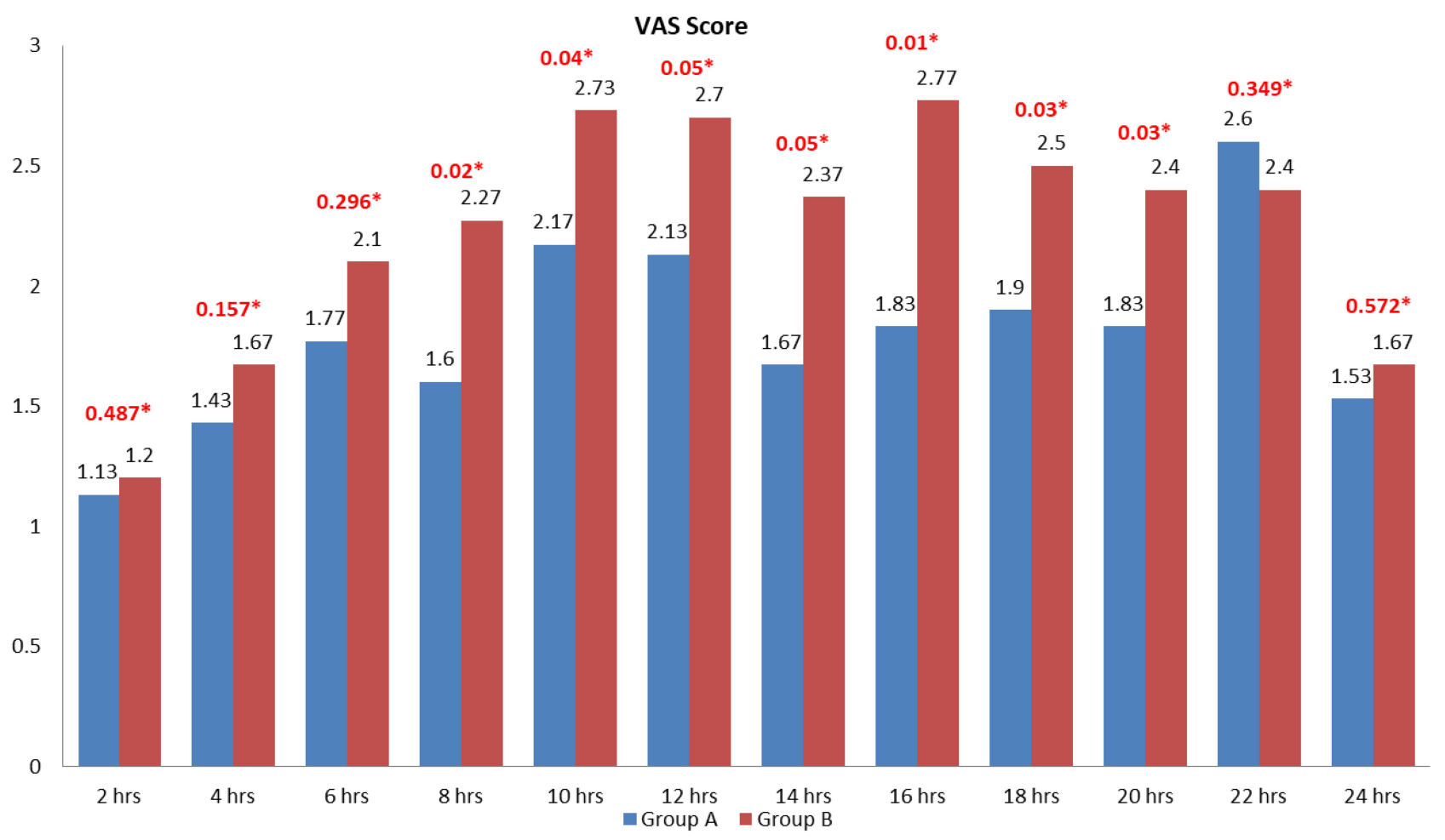

Figure 4 Visual analog scale scores.

Notes: Values are in mean. *P-value.

In the classical landmark technique, the entry point is bounded superiorly by the zygomatic arch and the mandibular arch, anterior and below the tragus of the ear. The needle is entered between the coronoid and condylar processes of the ramus of the mandible. The needle is entered in the space between the zygomatic arch and the center of the mandibular notch as high as possible. The needle penetrates the skin perpendicularly and advanced $2-4 \mathrm{~cm}$ towards the lateral pterygoid plate and then advanced posteriorly and inferiorly. After hitting the mandible, the needle is withdrawn and local anesthetics are injected after careful aspiration. ${ }^{16}$ The blind technique is associated with the risk of puncture of the internal maxillary or middle meningeal arteries. The use of ultrasound in the mandibular nerve block increases the success rate and reduces complications.

The mandibular nerve block is indicated for surgeries on the mandible including fractures and surgery on the lower lip and teeth. Though, a mandibular nerve block is associated with a higher success rate, it is surprisingly rarely used in anesthesia. The postoperative pain after faciomaxillary surgeries are often undertreated and poorly studied. The pain is usually poorly treated with paracetamol, non-steroidal anti-inflammatory drugs, and rarely with opioids. The use of regional anesthetic techniques may not only provide better analgesia but also improve rehabilitation. ${ }^{17}$

Morphine consumption was significantly reduced in group A than group B. The consumption was less than $6 \mathrm{mg}$ in both the groups showing the effectiveness of mandibular nerve block. The time for a request for rescue analgesic was also significantly prolonged in group A lasting for more than 13 hours than group B where it lasted for less than nine hours. There was a significant reduction in heart rate in group $A$ than group B intraoperatively lasting up to two hours after the block. There was no statistically significant reduction in mean arterial pressure between the two groups in spite of the values being less in group A than group B. The VAS score was also reduced in group A from $8 \mathrm{~h}$ lasting for $20 \mathrm{~h}$ postoperatively. There was no difference in VAS scores between the two groups for the first six hours due to the effect of the mandibular block. No complications were reported in both the groups signaling the safety of mandibular block.

Kumar et al performed a study on pre-emptive UG trigeminal nerve block in pterygopalatine fossa for faciomaxillary surgeries in 60 patients. They found that 
trigeminal nerve block reduces intraoperative consumption of fentanyl, postoperative consumption of opioids, and reduced pain scores postoperatively. They were able to demonstrate better pain scores for $24 \mathrm{~h}$ postoperatively ${ }^{18}$ The findings were similar were to our study but we blocked only the mandibular nerve and not the trigeminal nerve which may be associated with more complications and not required as well. Nader et al evaluated the efficacy of UG trigeminal nerve block for patients with facial pain. They were able to demonstrate complete sensory analgesia on one side of the face within $10 \mathrm{~min}$ of injection. They also did not find any neurological sequelae to trigeminal nerve block when patients were followed for 6-12 months. ${ }^{19}$ Van Lancker et al studied the efficacy of mandibular nerve block in mandibular osteotomy surgeries. The pre-emptive administration of block reduced the consumption of sufentanil intraoperatively and reduction in postoperative consumption of opioids and its adverse effects. $^{20}$ The results of this study correlate with our study. Plantevin et al conducted a study on the effects of mandibular nerve block for surgeries of oropharyngeal carcinoma for postoperative analgesia. They found that mandibular nerve block improved postoperative analgesia, reduced the incidence of severe pain and consumption of morphine postoperatively. ${ }^{21}$ This study highlights the efficacy of mandibular nerve block for tumor resection as well.

Krishnan et al evaluated the efficacy of pre-emptive with $0.25 \%$ bupivacaine for elective orthognathic surgery. The pre-incisional nerve blocks given depending on the type of surgery include posterior superior alveolar nerve block, infraorbital nerve block, and greater palatine nerve block for the maxillary procedures and mandibular nerve block and inferior alveolar nerve block for the mandible. All the blocks were administered using the classic landmark guided technique and compared with the control group. The study group patients had better VAS scores postoperatively and only a few patients required rescue analgesics when compared to the control group. They concluded that pre-emptive nerve blocks can be easily administered and reduces postoperative pain in oral and faciomaxillary surgeries. ${ }^{22}$ Shetty et al analyzed the effectiveness of regional nerve blocks given before bimaxillary surgery for postoperative pain. They pronounced that pre-emptive nerve blocks reduce postoperative discomfort, pain, and consumption of analgesics. ${ }^{23}$ These studies show the superiority of pre-emptive facial nerve blocks.

The limitation of the study was that we did not check the exact areas of the sensory blockade. We also did not check if the pre-induction administration of mandibular nerve block improved the mouth opening which may be restricted due to fracture pain. Also, we did not use it for parasymphyseal fracture and bilateral fractures where bilateral blockade may help. There is scope for future research on mandibular nerve blocks in oral neoplastic surgeries as well as chronic pain.

To conclude, ultrasound-guided mandibular nerve block given before incision reduces the consumption of morphine for $24 \mathrm{~h}$ postoperatively, prolongs the time for a request for rescue analgesic and reduces intraoperative fentanyl consumption, better control of intraoperative heart rate, and better pain scores postoperatively when compared to mandibular nerve block administered at the end of surgery.

\section{Data Sharing Statement}

The data used to support the findings of this study are available from the corresponding author upon request.

\section{Disclosure}

The authors report no conflicts of interest for this work.

\section{References}

1. Benyamin R, Trescot AM, Datta S, et al. Opioid complications and side effects. Pain Physician. 2008;11(2):105-120.

2. Venkatraman R, Saravanan R, Mohana KV, Pushparani A. Evaluation of ultrasound-guided quadratus lumborum block for post-operative analgesia in unilateral laparoscopic renal surgeries - a randomised controlled trial. Indian J Anaesth. 2020;64:1007-1011. doi:10.4103/ ija.IJA_335_20

3. Penprase B, Brunetto E, Dahmani E, Forthoffer JJ, Kapoor S. The efficacy of preemptive analgesia for postoperative pain control: a systematic review of the literature. AORN J. 2015;101(1):94-105. doi:10.1016/j.aorn.2014.01.030

4. Obata H, Saito S, Fujita N, Fuse Y, Ishizaki K, Goto F. Epidural block with mepivacaine before surgery reduces long-term post-thoracotomy pain. Can J Anaesth. 1999;46:1127-1132. doi:10.1007/BF03015520

5. Zhu X. Efficacy of preemptive analgesia versus postoperative analgesia of celecoxib on postoperative pain, patients' global assessment and hip function recovery in femoroacetabular impingement patients underwent hip arthroscopy surgery. Inflammopharmacology. 2020;28 (1):131-137. doi:10.1007/s10787-019-00648-8

6. Long JB, Bevil K, Giles DL. Preemptive analgesia in minimally invasive gynecologic surgery. J Minim Invasive Gynecol. 2019;26 (2):198-218. doi:10.1016/j.jmig.2018.07.018

7. Møiniche S, Kehlet H, Dahl JB. A qualitative and quantitative systematic review of preemptive analgesia for postoperative pain relief: the role of timing of analgesia. Anesthesiology. 2002;96(3):725-741. doi:10.1097/00000542-200203000-00032

8. Venkatraman R, Abhinaya RJ, Sakthivel A, Sivarajan G. Efficacy of ultrasound-guided transversus abdominis plane block for postoperative analgesia in patients undergoing inguinal hernia repair. Local Reg Anesth. 2016;9:7-12. doi:10.2147/LRA.S93673

9. Aglio LS, Abd-El-Barr MM, Orhurhu V, et al. Preemptive analgesia for postoperative pain relief in thoracolumbosacral spine operations: a double-blind, placebo-controlled randomized trial. J Neurosurg Spine. 2018;29(6):647-653. doi:10.3171/2018.5.SPINE171380 
10. Woolf CJ. Evidence for a central component of post injury pain hypersensitivity. Nature. 1983;308:386-388.

11. Kien NT, Geiger P, Van Chuong H, et al. Preemptive analgesia after lumbar spine surgery by pregabalin and celecoxib: a prospective study. Drug Des Devel Ther. 2019;13:2145-2152. doi:10.2147/ DDDT.S202410

12. Kurd MF, Kreitz T, Schroeder G, Vaccaro AR. The role of multimodal analgesia in spine surgery. $J$ Am Acad Orthop Surg. 2017;25 (4):260-268. doi:10.5435/JAAOS-D-16-00049

13. Byrne K, Smith C. Preemptive analgesia: an unobtainable goal? $J$ Cardiothorac Vasc Anesth. 2019;33(2):460-461. doi:10.1053/j. jvca.2018.08.008

14. Munro A, Sjaus A, George RB. Anesthesia and analgesia for gynecological surgery. Curr Opin Anaesthesiol. 2018;31(3):274-279. doi:10.1097/ACO.0000000000000584

15. Kissin I, Weiskopf R. Preemptive analgesia. Anesthesiology. 2000;93:1138-1143. doi:10.1097/00000542-200010000-00040

16. Nerve Blocks of the Face. NYSORA; 2021. Available from: https:// www.nysora.com/techniques/head-and-neck-blocks/nerve-blocksface. Accessed January 25, 2021.

17. Nguyen A, Girard F, Boudreault D, et al. Scalp nerve blocks decrease the severity of pain after craniotomy. Anesth Analg. 2001;93:1272-1276. doi:10.1097/00000539-200111000-00048
18. Kumar A, Sinha C, Kumar A, Kumari P, Mukul SK. Ultrasound-guided trigeminal nerve block and its comparison with conventional analgesics in patients undergoing faciomaxillary surgery: randomised control trial. Indian J Anaesth. 2018;62:871-875. doi:10.4103/ija.IJA_256_18

19. Nader A, Kendall M. Dexamethasone versus triamcinolone side effects for ultrasound-guided trigeminal nerve block for the treatment of refractory typical or atypical facial pain. J Pain. 2016;17:72. doi:10.1016/j.jpain.2016.01.367

20. Van Lancker P, Abeloos JV, De Clercq CA, Mommaerts MY. The effect of mandibular nerve block on opioid consumption, nausea and vomiting in bilateral mandibular osteotomies. Acta Anaesthesiol Belg. 2003;54:223-226.

21. Plantevin F, Pascal J, Morel J, et al. Effect of mandibular nerve block on postoperative analgesia in patients undergoing oropharyngeal carcinoma surgery under general anaesthesia. Br J Anaes. 2007;5:708-12.

22. Krishnan R, Shivananda S, Raman U. Pre-emptive analgesia for elective maxillofacial surgery using $0.25 \%$ bupivacaine. Indian $J$ Anaesth. 2008;52:556.

23. Shetty V, BhanuPrakash B, Yadav A, Kishore PN, Menon A. Do regional nerve blocks before bimaxillary surgery reduce postoperative pain? J Oral Maxillofac Surg. 2020;78(5):724-730. doi:10.1016/j.joms.2019.10.014

\section{Publish your work in this journal}

Local and Regional Anesthesia is an international, peer-reviewed, open access journal publishing on the development, pharmacology, delivery and targeting and clinical use of local and regional anesthetics and analgesics. The journal welcomes submitted papers covering original research, basic science, clinical studies, reviews \& evaluations, guidelines, expert opinion and commentary, case reports and extended reports. The manuscript management system is completely online and includes a very quick and fair peer-review system, which is all easy to use. Visit http://www.dovepress.com/testimonials. php to read real quotes from published authors. 